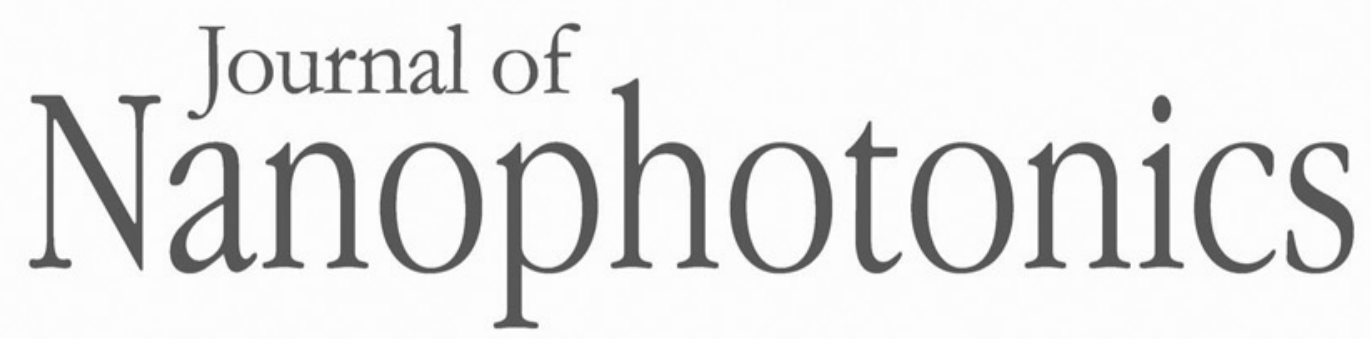

Nanophotonics.SPIEDigitalLibrary.org

\title{
Special Section Guest Editorial: Nanophotonic Materials and Devices
}

\author{
Maria-Pilar Bernal \\ Chii-Chang Chen \\ Chengkuo Lee
}

\section{SPIE.}




\title{
Special Section Guest Editorial: Nanophotonic Materials and Devices
}

\author{
Maria-Pilar Bernal, ${ }^{\mathrm{a}, *}$ Chii-Chang Chen, ${ }^{\mathrm{b}, *}$ and Chengkuo Lee ${ }^{\mathrm{c}, *}$ \\ ${ }^{a}$ FEMTO-ST, Optics Department, UMR 6174, CNRS, 16 Route de Gray, \\ 25030 Besancon, France \\ bNational Central University, Department of Optics and Photonics, 300 Chung-Da Road, \\ 320 Chung-Li, Taiwan \\ ${ }^{\mathrm{c}}$ National University of Singapore, Department of Electrical \& Computer Engineering, \\ Singapore 117576, Singapore
}

Nanophotonic technology is used to control the behaviors of light by using nanostructures. Owing to the progress of the fabrication techniques of nanostructures during the past decades, nanophotonics has become a study that can be investigated experimentally. It has been applied in several domains, including display, lighting, solar cells, and biophotonics, to obtain some new optical properties or better performances. Many novel interesting topics also have appeared recently, such as nanoantenna, metamaterials, plasmonics, etc. These results are changing our thinking about classical optics.

The Optics \& Photonics Taiwan International Conference (OPTIC) 2013 was held at National Central University (Chung-Li, Taiwan) on 5th-7th December 2013. Conference I: Nanophotonic Materials and Devices of OPTIC 2013 was devoted to fundamental nanophotonic properties and applications of nanomaterials or nanostructured materials. Based on the proceeding of the conference, this special section mainly contributes to state-of-the-art of nanophotonic materials and devices. These full-length papers provide a detailed discussion of the abstracts in the proceeding and report the development of the nanophotonic devices using novel nanophotonic materials.

The guest editors thank all of the authors for their contributions to this special section, the reviewers, editorial committee, especially Dr. M.-P. Bernal for her contribution, and all those who participated in the Conference I: Nanophotonic Materials and Devices of OPTIC 2013 and contributed to its proceedings.

\footnotetext{
*Address all correspondence to: Maria-Pilar Bernal, E-mail: maria-pilar.bernal@univ-fcomte.fr; Chii-Chang Chen, E-mail: trich@dop.ncu.edu.tw; and Chengkuo Lee, E-mail: elelc@nus.edu.sg
} 